\title{
Diagnosis of non-alcoholic fatty liver disease (NAFLD)
}

\author{
Hannele Yki-Järvinen ${ }^{1,2}$
}

Received: 11 January 2016 / Accepted: 23 February 2016/Published online: 18 April 2016

(C) Springer-Verlag Berlin Heidelberg 2016

\begin{abstract}
Non-alcoholic fatty liver disease (NAFLD) increases risk of mortality from liver and cardiovascular disease (CVD) and is the major cause of hepatocellular carcinoma (HCC), which may develop without cirrhosis. NAFLD predicts type 2 diabetes, even independently of obesity. Globally, the prevalence of NAFLD averages $25 \%$ and is as common as the metabolic syndrome. The majority of patients with type 2 diabetes have NAFLD. The challenge for the diabetologist is to identify patients at risk of advanced liver disease and HCC. At a minimum, liver function tests (LFTs), despite being neither specific nor sensitive, should be performed in all patients with the metabolic syndrome or type 2 diabetes. Increases in LFTs, for which the updated reference values are lower (serum $\mathrm{ALT} \approx 30 \mathrm{U} / 1$ in men and $\approx 20 \mathrm{U} / 1$ in women) than those hitherto used in many laboratories, should prompt assessment of fibrosis biomarkers and referral of individuals at risk to a NAFLD/hepatology clinic. Preferably, evaluation of NAFLD should be based on measurement of steatosis biomarkers or ultrasound if easily available. A large number of individuals carry the patatin-like phospholipase domain containing 3 (PNPLA3) I148M variant (30-50\%) or the transmembrane 6 superfamily member 2 (TM6SF2) E167K variant $(11-15 \%)$. These variants increase the risk of advanced liver disease and HCC but not of diabetes or CVD. Genotyping of selected patients for these variants is recommended. Many patients have 'double trouble', i.e. carry
\end{abstract}

Hannele Yki-Järvinen

Hannele.Yki-Jarvinen@helsinki.fi

1 Department of Medicine, University of Helsinki, Haartmaninkatu 8, 00290 Helsinki, Finland

2 Minerva Foundation Institute for Medical Research, Helsinki, Finland both a genetic risk factor and have the metabolic syndrome. Excess use of alcohol could be a cause of 'triple trouble', but such patients would be classified as having alcoholic fatty liver disease. This review summarises a presentation given at the symposium 'The liver in focus' at the 2015 annual meeting of the EASD. It is accompanied by two other reviews on topics from this symposium (by Kenneth Cusi, DOI: 10. 1007/s00125-016-3952-1, and by John Jones, DOI: 10.1007/ s00125-016-3940-5) and a commentary by the Session Chair, Michael Roden (DOI: 10.1007/s00125-016-3911-x).

Keywords Alanine aminotransferase · Diabetes · Insulin . Metabolic syndrome $\cdot$ PNPLA3 (patatin-like phospholipase domain containing 3) $\cdot$ Review $\cdot$ Steatosis $\cdot$ TM6SF2 (transmembrane 6 superfamily member 2 ) · Triacylglycerols * Ultrasound
Abbreviations
ALT Alanine aminotransferase
AST Aspartate aminotransferase
CVD Cardiovascular disease
EASD European Association for the Study of Diabetes
EASL European Association for the Study of the Liver
EASO European Association for the Study of Obesity
GGT $\quad \gamma$-Glutamyl transpeptidase
HCC Hepatocellular carcinoma
$\mathrm{HCV} \quad$ Hepatitis $\mathrm{C}$ virus
${ }^{1} \mathrm{H}-\mathrm{MRS} \quad$ Proton magnetic resonance spectroscopy
LFTs Liver function tests
NAFL Non-alcoholic fatty liver
NAFLD Non-alcoholic fatty liver disease
NASH Non-alcoholic steatohepatitis
PNPLA3 Patatin-like phospholipase domain containing 3
TE Transient elastography 
TM6SF2 Transmembrane 6 superfamily member 2 US Ultrasound

\section{Introduction}

Non-alcoholic fatty liver disease (NAFLD) is defined as a condition in which $\geq 5-10 \%$ of hepatocytes exhibit macroscopic steatosis by light microscopy in the absence of other aetiologies of liver disease (see below) [1,2]. NAFLD includes a spectrum of liver disease ranging from simple steatosis (non-alcoholic fatty liver [NAFL]) to non-alcoholic steatohepatitis (NASH). NALFD is by far the most common cause of chronic liver disease. In a recent meta-analysis, the global prevalence was estimated to be $25 \%$ [2], which is very similar to that of the metabolic syndrome [3]. Prevalence estimates averaged $32 \%$ in the Middle East, $30 \%$ in South America, $27 \%$ in Asia, 24\% in Europe, $21 \%$ in North America and $13 \%$ in Africa in a meta-analysis conducted in 2015 [2]. Such NAFLD associated with features of the metabolic syndrome predisposes individuals to type 2 diabetes and cardiovascular disease (CVD), as well to NASH, cirrhosis and hepatocellular carcinoma (HCC) and the need for liver transplantation $[4,5]$. NAFLD and the metabolic syndrome share common pathophysiology, which is why they frequently coexist. This is not uniformly true, however, in part because of the multiple definitions of the metabolic syndrome, and because of common genetic forms of NAFLD not associated with insulin resistance. The most recent recommendation allows diagnosis of the metabolic syndrome in ten different ways. The metabolic syndrome is defined as a condition that includes any three of the following five conditions: increased fasting glucose or type 2 diabetes, hypertriacylglycerolaemia, low HDL-cholesterol, increased waist circumference (ethnicity-dependent) and hypertension [6].

Common genetic forms of NAFLD, especially those associated with variation in the genes PNPLA3 and TM6SF2 are not associated with insulin resistance, features of the metabolic syndrome or an increased risk of type 2 diabetes or CVD [5]. However, in these patients, NAFLD predisposes to progression of NAFL to NASH, cirrhosis and HCC. The ensuing discussion is focused on the diagnosis of NAFLD from the perspective of the diabetologist. The challenge for the diabetologist is to identify those at risk for advanced liver disease amongst those who have incidentally discovered steatosis and those with no such history.

\section{Definitions}

The diagnosis of NAFLD requires that there is evidence of steatosis either by imaging or histology and that there are no secondary causes of steatosis, such as increased alcohol consumption, viral hepatitis, use of steroid medications or other causes, as listed in Fig. 1 and more extensively in [7].

NAFL Steatosis can be diagnosed either using a liver biopsy ( $\geq 5-10 \%$ of hepatocytes exhibit macroscopic steatosis) or by imaging techniques such as ultrasound (US) (degree of brightness of liver parenchyma, semi-quantitative) or proton magnetic resonance spectroscopy $\left({ }^{1} \mathrm{H}-\mathrm{MRS}\right)$ [8]. Steatosis on US and ${ }^{1} \mathrm{H}$-MRS does not, however, exclude $\mathrm{NASH}$, as they merely quantify steatosis. Histologically, NAFL encompasses any degree of steatosis alone or steatosis with lobular inflammation but without ballooning [9].

NASH NASH can only be diagnosed by liver biopsy. The presence of ballooning injury is the key to the diagnosis. Ballooning degeneration is a form of hepatocyte cell death where the cells increase in cell size (balloon). It colocalises initially amidst steatosis in zone 3 near the centrilobular veins. Steatosis and inflammation can be observed to any degree [9]. Fibrosis is not required to make the diagnosis of NASH but is often present. It also begins in zone 3 as delicate strands of collagen [9]. Recently, a new definition based on the SAF score, which does not subclassify patients with NAFLD based on NASH, has been proposed [10]. The SAF score assesses three variables: $\mathrm{S}=$ steatosis, $\mathrm{A}=$ activity, $\mathrm{F}=$ fibrosis. Steatosis $(\%$ of hepatocytes with macroscopic steatosis) is classified on a scale of 0 to 3 (S0: $<5 \%, \mathrm{~S} 1$ : 5-33\%, S2: $34-66 \%, \mathrm{~S} 3:>67 \%$ ). Ballooning and lobular inflammation are graded from 0 to 2 . The grade of activity (from A0 to A4) is calculated by addition of grades of ballooning and lobular inflammation [10]. Fibrosis $(\mathrm{F})$ is scored on a scale of 0 to 4 (F0: as none, F1: perisinusoidal or periportal, F2: perisinusoidal and periportal, F3: bridging or F4: cirrhosis [11]. This scoring system has been shown to decrease intra-observer variation among pathologists [10]. It is also attractive as it includes the fibrosis component which is not required for diagnosis of NASH but is the best predictor of advanced liver disease [12] and mortality [13].

\section{Why should a diabetologist be interested in NAFLD?}

NAFLD is common in type 2 diabetes The 95th percentile of normal liver fat in 2,349 participants in the population-based Dallas Heart Study was $5.56 \%$ when measured using ${ }^{1} \mathrm{H}-\mathrm{MRS}$ [8]. Using this technique and criterion, the prevalence of NAFLD in type 2 diabetic patients has been reported to be $50 \%$ [14] and $60 \%$ [15]. A study diagnosing NAFLD by MRIestimated proton density fat fraction $\geq 5 \%$ found NAFLD in $65 \%$ [16] and studies using ultrasound diagnosed NAFLD in $74 \%$ [17], 57\% [18] and 70\% [19] of patients with type 2 diabetes. Half of patients with type 2 diabetes have NAFLD despite having normal alanine aminotransferase (ALT) levels 
Incidental steatosis

(US/CT/MRI)

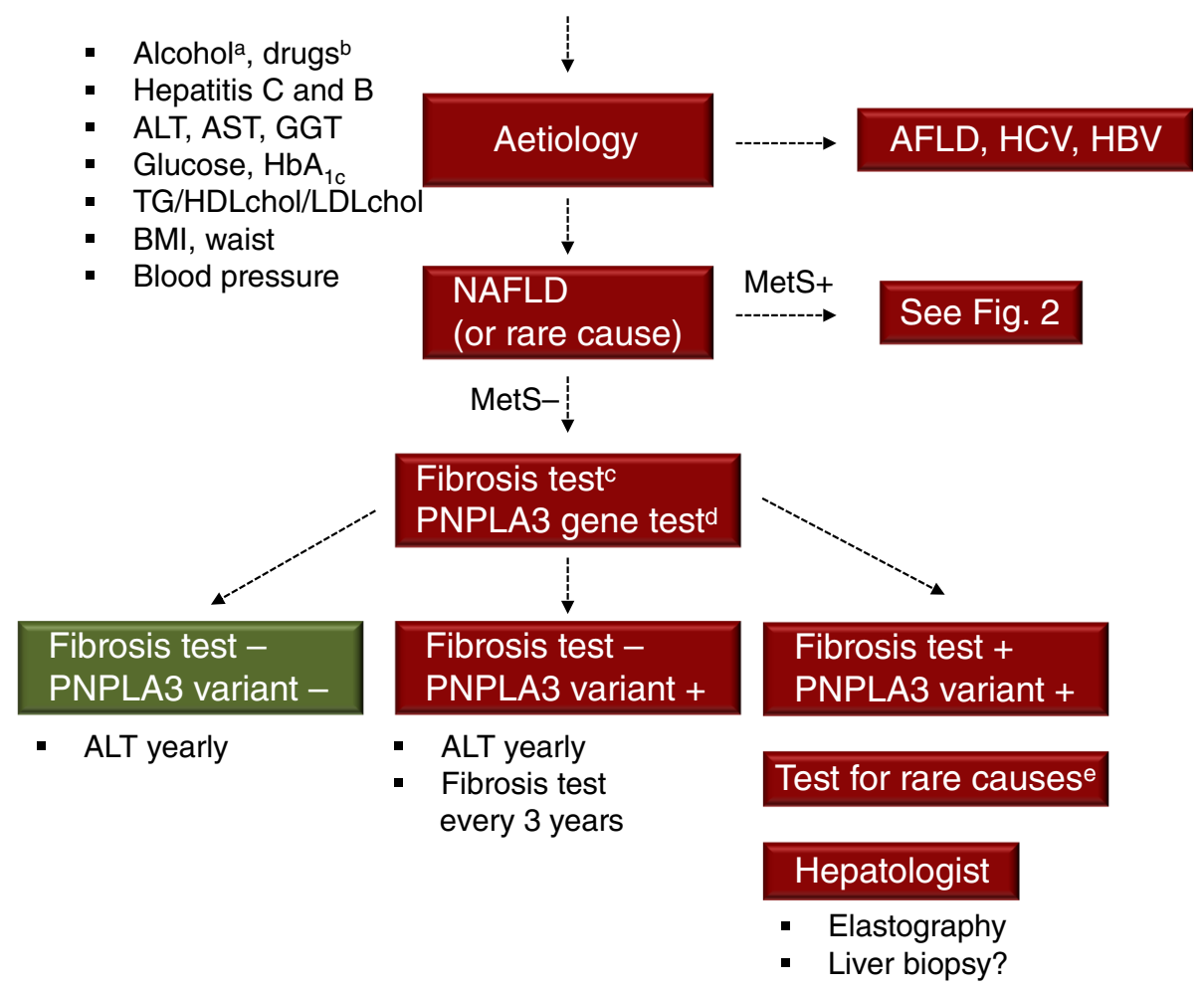

Fig. 1 Evaluation of incidentally discovered steatosis. Alcohola ${ }^{\text {: }}$ for diagnosis of NAFLD, alcohol consumption should be less than $20 \mathrm{~g}$ /day in women and less than $30 \mathrm{~g} /$ day in men. Drugs ${ }^{\mathrm{b}}$ : these include medications such as corticosteroids, methotrexate, amiodarone and tamoxifen. Fibrosis test ${ }^{\mathrm{c}}$ refers to use of fibrosis biomarkers such as the NAFLD fibrosis score, Fibrotest, FibroMeter or ELF. PNPLA3 gene test ${ }^{\mathrm{d}}$ : genotyping for the I148M gene variant. PNPLA3 variant +: carrier of the I148M gene variant. PNPLA3 variant -: non-carrier of the I148M gene variant. Fibrosis test + : moderate or high risk for fibrosis, biomarker-dependent. Fibrosis test -: low risk for fibrosis. ${ }^{\mathrm{e}}$ Tests for rare causes of steatosis and

[14]. Regarding NASH, a population-based study using a NASH score validated in 296 Finnish and 380 Italian individuals who had undergone a liver biopsy, determined the prevalence of NASH to be $17.6 \%$ in type 2 diabetic individuals and $3.7 \%$ in non-diabetic individuals [20]. In a biopsy study, $22 \%$ of type 2 diabetic patients attending an outpatient clinic were found to have NASH [17]. Advanced fibrosis, as measured by magnetic resonance elastography, was found in $7 \%$ of type 2 diabetic patients [16].

NAFLD predicts type 2 diabetes and cardiovascular disease At least 20 prospective studies have shown that steatosis diagnosed by US or biopsy predicts NAFLD, even independent of obesity [4]. This is reminiscent of the metabolic syndrome, which can occur in non-obese people and predicts type 2 diabetes and CVD even independent of obesity [5]. Indeed, one of the first definitions of the metabolic syndrome, liver disease in general need to be examined before referral to the specialist (depends on local practices and clinical judgement) and include tests such as those for autoimmune, coeliac disease, thyroid disease, Wilson's disease, $\alpha 1$-antitrypsin deficiency and measurement of ferritin and transferrin saturation, and total and conjugated bilirubin and thromboplastin time. Abbreviations: AFLD, alcoholic fatty liver disease; ALT, alanine aminotransferase; $\mathrm{CT}$, computerised tomography; $\mathrm{HBV}$, steatosis due to hepatitis $\mathrm{B}$ virus; $\mathrm{HCV}$, steatosis due to hepatitis $\mathrm{C}$ virus; HDLchol, HDL cholesterol; LDLchol, LDL cholesterol; waist, waist circumference; MetS, metabolic syndrome; TG, triacylglycerol

proposed by Reaven, did not even include obesity [21]. Nevertheless, obesity remains the single most important risk factor for NAFLD [22]. Insulin resistance in individuals with the metabolic syndrome is paralleled by an increase in liver fat content and serum ALT and aspartate aminotransferase (AST) activities [23, 24]. Insulin resistance is characterised by an impaired ability of insulin to suppress hepatic glucose production. The increase in glucose concentrations increases insulin secretion, thus resulting in mild hyperglycaemia and hyperinsulinaemia - hallmarks of insulin resistance and NAFLD [5]. Other components of the metabolic syndrome are also closely related. Impaired insulin inhibition of VLDL production leads to hypertriacylglycerolaemia, which in turn reduces HDL-cholesterol concentrations [5].

Fibrosis the best predictor of progression A recent longitudinal study reported that the fibrosis stage of all features of 
liver histology increased the risk of overall mortality and liver transplantation in 619 NAFLD patients followed for 12.6 years [12]. In a smaller study involving 229 Swedish patients with histologically diagnosed NAFLD followed for 26.4 years, advanced fibrosis also increased mortality independently of other histological features [13]. Based on the observed annual fibrosis progression rate, a meta-analysis estimated that one stage progression takes 14 years for patients with NAFL and 7 years for patients with NASH [25].

NAFL can progress to NASH McPherson et al recently concluded 'Contrary to current dogma ... steatosis can progress to NASH and clinically significant fibrosis' [26]. In their study, 27 patients from a NAFLD clinic in the UK with baseline NAFL underwent two biopsies, one at baseline and one after an average of 6.6 years. Progression to NASH was seen in $44 \%$ [26]. Similarly, a French study followed 25 patients with NAFL for 3.7 years and found that in a substantial proportion of patients with NAFL (32\%) the disease clearly progressed to NASH [27]. Of 13 patients with NAFL from Hong Kong, $23 \%$ developed definite NASH over a 3 -year follow-up period [28].

NAFLD is the most common cause of HCC In a prospectively studied population of more than 900,000 adults in the USA, of all the cancers, obesity increased the relative risk of liver cancer the most, especially in men [29]. An association between obesity and HCC has also been observed in large epidemiological cohorts in Korea, Sweden, Norway and Austria, amongst others [30-32]. A study in an Italian cohort provided evidence that the risk of HCC increases progressively as a function of the number of components of the metabolic syndrome [33].

Diabetes increases the risk of HCC by two- to threefold [34]. In a series of 632 consecutive patients attending a hepatology clinic, the increase in HCC over 10 years paralleled the increase in NAFLD, which accounted for $35 \%$ of the HCC cases in 2010 and was the most common cause of HCC [35]. Worryingly, HCC may occur in NAFLD patients without cirrhosis $[35,36]$. These data are of major concern and emphasise the need to develop effective screening tools to enable the early diagnosis of HCC in patients with obesity, NAFLD, the metabolic syndrome or type 2 diabetes.

\section{Diagnosis of NAFLD in a patient with incidentally discovered steatosis}

The European Associations for the Study of the Liver (EASL), Diabetes (EASD) and Obesity (EASO) have recently worked together on Clinical Practice Guidelines for the management of NAFLD [7]. According to these guidelines, all individuals with incidentally discovered steatosis should be screened for features of the metabolic syndrome, independently of liver function tests (LFTs). For diagnosis of NAFLD, other causes of steatosis should be excluded (see Fig. 1). These include, at a minimum, alcohol, hepatitis B and C and steatosis-associated drugs. Components of the metabolic syndrome and diabetes should be screened for using tools familiar to the diabetologist (see Fig. 1), and the screening includes assessment of dietary and lifestyle habits. As regards diet, in addition to total energy intake, particular emphasis should be paid to consumption of simple sugars and saturated fats, both of which are important risk factors for obesity and, independently of obesity for type 2 diabetes, CVD and NAFLD [7, 37]. In addition, LFTs, platelets and albumin should be measured to enable calculation of a non-invasive score for NAFLD, such as the NAFLD fibrosis score (based on age, BMI, impaired fasting glucose/diabetes, AST, ALT, platelets, albumin; calculator found at www. nafldscore.com). Patients with increased LFTs in addition to steatosis and those with normal LFTs but a fibrosis biomarker indicating medium/high risk should be referred to the hepatologist (Fig. 2). The cost-benefit of this recommendation needs to be investigated [7].

The hepatologist will usually perform an US examination, unless this has already been done, and transient elastography (TE) to exclude severe fibrosis and assess the need for a liver biopsy. TE is a quick, non-invasive technique for measuring liver stiffness. A probe with an ultrasonic transducer is placed above the liver. It generates an elastic shear wave, the velocity of which is related to liver stiffness and fibrosis [38]. TE is sensitive and specific in excluding advanced stage 3-4 fibrosis but fails in approximately $20 \%$, mainly due to obesity [38]. Diagnosis of NASH still requires a liver biopsy [7]. In patients with steatosis who initially have normal liver enzymes and low fibrosis biomarkers, these measurements should be repeated every 2 years.

\section{Screening for NAFLD in a patient with the metabolic syndrome/type 2 diabetes}

The EASL-EASD-EASO NAFLD guidelines recommend screening for NAFLD in all patients with the metabolic syndrome or type 2 diabetes using at least LFTs but also steatosis biomarkers such as the NAFLD liver fat score based on knowledge of the metabolic syndrome, type 2 diabetes, fasting serum insulin, AST, AST/ALT (tool to calculate available in [39]), the Fatty Liver Index (waist, triacylglycerols and $\gamma$ glutamyl transpeptidase [GGT]) or Steatotest ${ }^{\circledR}$ (a commercially available test, undisclosed formula) [7]. US is recommended as an alternative tool if easily available, although the costeffectiveness of such a recommendation remains to be determined. Even if not cost-effective, US might help the early diagnosis of HCC. If liver enzymes are normal in patients with 


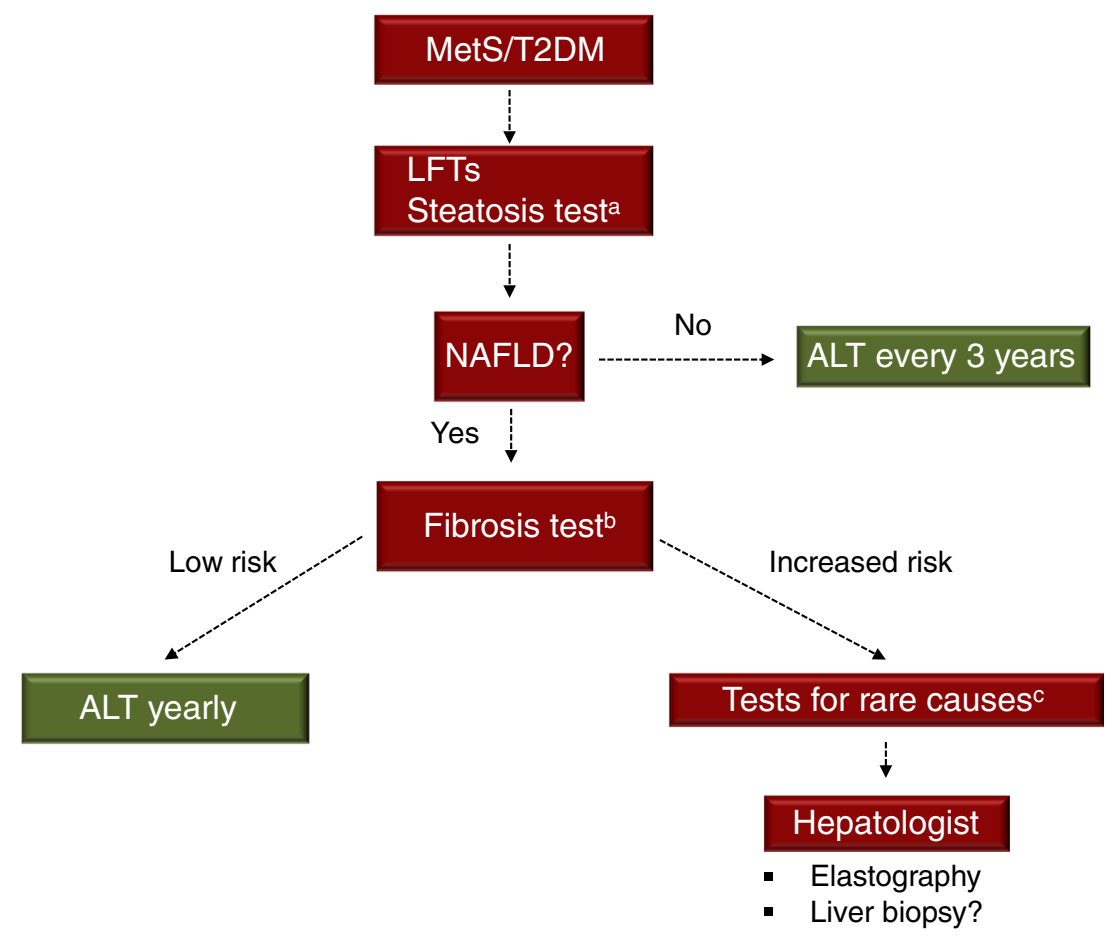

Fig. 2 Diagnosis of non-alcoholic fatty liver disease (NAFLD) in patients with the metabolic syndrome (MetS) or type 2 diabetes (T2DM). Screening for NAFLD by liver function tests (LFTs i.e. alanine aminotransferase [ALT], aspartate aminotransferase [AST], $\gamma$ glutamyltransferase [GGT]) and steatosis tests ${ }^{\mathrm{a}}$ such as the NAFLD liver fat score based on knowledge of the MetS, type 2 diabetes, fasting serum

the metabolic syndrome/NAFLD, repeat measurements are recommended every 3-5 years.

In patients who have been found to have NAFLD but have normal liver enzymes, the recommendation is to assess fibrosis tests and proceed as with patients with incidentally discovered steatosis. Lifestyle advice and comprehensive assessment of risk factors for CVD is essential in all patients with the metabolic syndrome/type 2 diabetes, or obesity alone.

The EASL-EASD-EASO guidelines recommend that all patients with elevated LFTs because of NAFLD should be referred to the hepatologist (Fig. 2), although LFTs are normal in $50 \%$ of patients with NAFLD [40] and type 2 diabetes [14]. Regarding normal values for LFTs, ALT is the most liverspecific, although ALT activities decrease and the AST:ALT ratio increases as fibrosis progresses [41, 42]. The increasing availability of standardised commercial assays is thought to greatly reduce the dependency of ALT results on the analytical procedure used and the need for separate reference intervals [41]. The upper limit of normal for ALT in many laboratories is around $40 \mathrm{IU} / 1$ in men and around $30 \mathrm{IU} / 1$ in women [41, 43]. Several of the previous and still currently used reference ranges for ALT were established in the 1980s before tests for hepatitis $\mathrm{C}$ virus (HCV) were available and before awareness of NAFLD [41]. More recent studies have established normal values that exclude all causes of viral hepatitis and individuals insulin, AST, AST/ALT (tool to calculate available in [39]), the fatty liver index (waist, triacylglycerol and GGT) or Steatotest (commercially available test, undisclosed formula) [7] should be part of routine work-up for patients with the MetS/T2DM [7]. Explanations for fibrosis test ${ }^{b}$ and tests for rare causes ${ }^{\mathrm{c}}$ as in legend to Fig. 1. Modified with permission from [7]

with steatosis and abnormal lipid and glucose levels. Updated upper limits of normal (95th or 97.5th percentile according to sex) have been proposed. These include $30 \mathrm{IU} / 1$ for men and $19 \mathrm{IU} / 1$ for women among Italians, 35 and $26 \mathrm{IU} / 1$ among Koreans, 21 and 17 IU/1 among Taiwanese and 35 and $23 \mathrm{IU} / 1$ among Chinese, respectively [41].

\section{Heterogeneity of NAFLD: NAFLD but no metabolic syndrome}

An allele in PNPLA3 (rs738409[G], encoding I148M) is found in $30-50 \%$ of all individuals. This gene variant has been shown, in numerous independent studies and meta-analyses, to confer an increased risk of steatosis, NASH, fibrosis, cirrhosis and HCC. Consistently, however, the gene variant does not predispose individuals to type 2 diabetes or CVD [5].

Patatin-like phospholipase domain containing 3 (PNPLA3) has both lipase and acylglycerol $O$-acyltransferase activities in vitro [44]. The I148M variant abolishes lipolysis [45] and increases lysophosphatidic acid acyltransferase activity [46], i.e. it both impairs breakdown and stimulates synthesis of triacylglycerols in the liver cells. In vivo in the human liver, the fatty acid composition of triacylglycerols and NEFA of individuals with the I148M variant is markedly polyunsaturated in 
contrast to 'obese/metabolic NAFLD', where saturated and monounsaturated triacylglycerols and NEFA predominate [47]. Insulin resistance inducing ceramides synthesised by the de novo ceramide synthetic pathway are increased in 'obese/metabolic NAFLD' but not in NAFLD associated with the I148M variant ('PNPLA3 NAFLD'). Lack of insulin resistance is likely to explain why this type of NAFLD does not predispose to type 2 diabetes and CVD.

Two independent studies reported the TM6SF2 gene to influence hepatic triacylglycerol secretion $[48,49]$. In carriers of the transmembrane 6 superfamily member 2 (TM6SF2) E167K variant $(11-15 \%$ of all individuals), the risk of steatosis [48], and fibrosis [50, 51] and HCC [52] are increased while the risk of CVD is even decreased [51]. Serum triacylglycerol and LDL-cholesterol are lower and insulin sensitivity unaltered $[48,51,53]$.

Both of the common genetic forms of NAFLD thus increase the risk of liver disease but not of type 2 diabetes and CVD. This implies that NAFLD is heterogeneous and that the aetiology of NAFLD has implications for risk of future disease. The EASL-EASD-EASO recommendation is to genotype for the PNPLA3 and TM6SF2 gene variants in selected patients and clinical studies but not routinely [7]. Such selected patients might include those who have NAFLD but do not have dyslipidaemia or insulin resistance. Given that both the gene variants and obesity/metabolic syndrome are common, many patients of course have 'double trouble' [47].

\section{Conclusions}

After years of focusing on the metabolic syndrome as the risk factor to search for without paying much attention to the liver, it is now clear that the metabolic syndrome and NAFLD frequently coexist because of a shared pathophysiology and that NASH, cirrhosis and hepatocellular carcinoma are more frequent in patients with the metabolic syndrome and type 2 diabetes than in those without. NAFL could thus be considered an essential part of the pathogenesis of the metabolic syndrome/type 2 diabetes and NASH, cirrhosis and even HCC complications of obesity/ the metabolic syndrome/type 2 diabetes. Patients with steatosis and the metabolic syndrome/type 2 diabetes therefore should be referred to the hepatologist more often than in the past, although the cost-effectiveness of referring all patients with elevated LFTs and the metabolic syndrome/type 2 diabetes still needs to be examined. The common genetic forms caused by polymorphisms in PNPLA3 and TM6SF2 genes predispose to liver disease but not to the metabolic syndrome/type 2 diabetes and CVD. This implies that measurement of components of the metabolic syndrome remains essential for identifying individuals at risk for type 2 diabetes and CVD. However, the repertoire of laboratory tests should include measurement of LFTs (ALT, AST and GGT), albumin and platelets to calculate the NAFLD fibrosis score or some other non-invasive marker of fibrosis. Given that NAFLD has become the leading cause of HCC and that HCC increases at alarming rates, screening of the liver by US more often than perhaps financially justified might be of benefit to the individual patient.

Acknowledgements I am grateful to all members of the group chaired by Giulio Marchesini that produced the EASL-EASD-EASO Clinical Practice Guidelines for the management of non-alcoholic fatty liver disease [7].

Funding Work by the author is supported by research grants from the Academy of Finland (HY), EU H2020 EPoS 634413 (HY), the Sigrid Juselius (HY), EVO (HY), and the EU/EFPIA Innovative Medicines Initiative Joint Undertaking (EMIF grant no. 115372, HY).

Duality of interest The author declares that there is no duality of interest associated with this manuscript.

Contribution statement The author was the sole contributor to the paper.

\section{References}

1. Neuschwander-Tetri BA, Caldwell SH (2003) Nonalcoholic steatohepatitis: summary of an AASLD Single Topic Conference. Hepatology 37:1202-1219

2. Younossi ZM, Koenig AB, Abdelatif D, Fazel Y, Henry L, Wymer M (2015) Global epidemiology of non-alcoholic fatty liver diseasemeta-analytic assessment of prevalence, incidence and outcomes. Hepatology

3. International Diabetes Federation (2006) The IDF consensus worldwide definition of the metabolic syndrome. International Diabetes Federation, Brussels

4. Anstee QM, Targher G, Day CP (2013) Progression of NAFLD to diabetes mellitus, cardiovascular disease or cirrhosis. Nat Rev Gastroenterol Hepatol 10:330-344

5. Yki-Jarvinen H (2014) Non-alcoholic fatty liver disease as a cause and a consequence of metabolic syndrome. Lancet Diabetes Endocrinol 2:901-910

6. Alberti KG, Eckel RH, Grundy SM et al (2009) Harmonizing the metabolic syndrome: a joint interim statement of the International Diabetes Federation Task Force on Epidemiology and Prevention; National Heart, Lung, and Blood Institute; American Heart Association; World Heart Federation; International Atherosclerosis Society; and International Association for the Study of Obesity. Circulation 120:1640-1645

7. European Association for the Study of the Liver (EASL), European Association for the Study of Diabetes (EASD) and European Association for the Study of Obesity (EASO) (2016) EASLEASD-EASO clinical practice guidelines for the management of non-alcoholic fatty liver disease. J Hepatol doi:10.1016/j.jhep. 2015.11.004

8. Szczepaniak LS, Nurenberg P, Leonard D et al (2005) Magnetic resonance spectroscopy to measure hepatic triglyceride content: prevalence of hepatic steatosis in the general population. Am J Phys Endocrinol Metab 288:E462-E468

9. Kleiner DE, Brunt EM (2012) Nonalcoholic fatty liver disease: pathologic patterns and biopsy evaluation in clinical research. Semin Liver Dis 32:3-13 
10. Bedossa P, Consortium FP (2014) Utility and appropriateness of the fatty liver inhibition of progression (FLIP) algorithm and steatosis, activity, and fibrosis (SAF) score in the evaluation of biopsies of nonalcoholic fatty liver disease. Hepatology 60:565-575

11. Kleiner DE, Brunt EM, Van Natta M et al (2005) Design and validation of a histological scoring system for nonalcoholic fatty liver disease. Hepatology 41:1313-1321

12. Angulo P, Kleiner DE, Dam-Larsen S et al (2015) Liver fibrosis, but no other histologic features, is associated with long-term outcomes of patients with nonalcoholic fatty liver disease. Gastroenterology 149(389-397):e310

13. Ekstedt M, Hagstrom H, Nasr P et al (2015) Fibrosis stage is the strongest predictor for disease-specific mortality in NAFLD after up to 33 years of follow-up. Hepatology 61:1547-1554

14. Portillo-Sanchez P, Bril F, Maximos M et al (2015) High prevalence of nonalcoholic fatty liver disease in patients with type 2 diabetes mellitus and normal plasma aminotransferase levels. J Clin Endocrinol Metab 100:2231-2238

15. Petit JM, Guiu B, Terriat B et al (2009) Nonalcoholic fatty liver is not associated with carotid intima-media thickness in type 2 diabetic patients. J Clin Endocrinol Metab 94:4103-4106

16. Doycheva I, Cui J, Nguyen P et al (2016) Non-invasive screening of diabetics in primary care for NAFLD and advanced fibrosis by MRI and MRE. Aliment Pharmacol Ther 43:83-95

17. Williams CD, Stengel J, Asike MI et al (2011) Prevalence of nonalcoholic fatty liver disease and nonalcoholic steatohepatitis among a largely middle-aged population utilizing ultrasound and liver biopsy: a prospective study. Gastroenterology 140:124-131

18. Williamson RM, Price JF, Glancy S et al (2011) Prevalence of and risk factors for hepatic steatosis and nonalcoholic Fatty liver disease in people with type 2 diabetes: the Edinburgh Type 2 Diabetes Study. Diabetes Care 34:1139-1144

19. Targher G, Bertolini L, Padovani R et al (2007) Prevalence of nonalcoholic fatty liver disease and its association with cardiovascular disease among type 2 diabetic patients. Diabetes Care 30:12121218

20. Hyysalo J, Mannisto VT, Zhou Y, et al. (2013) A population-based study on the prevalence of NASH using scores validated against liver histology. J Hepatol 60:839-846

21. Reaven GM (1988) Banting lecture 1988. Role of insulin resistance in human disease. Diabetes 37:1595-1607

22. Lazo M, Hernaez R, Eberhardt MS et al (2013) Prevalence of nonalcoholic fatty liver disease in the United States: the third national health and nutrition examination survey, 1988-1994. Am J Epidemiol 178:38-45

23. Seppala-Lindroos A, Vehkavaara S, Hakkinen AM et al (2002) Fat accumulation in the liver is associated with defects in insulin suppression of glucose production and serum free fatty acids independent of obesity in normal men. J Clin Endocrinol Metab 87:30233028

24. Kotronen A, Yki-Jarvinen H (2008) Fatty liver: a novel component of the metabolic syndrome. Arterioscler Thromb Vasc Biol 28:2738

25. Singh S, Allen AM, Wang Z, Prokop LJ, Murad MH, Loomba R (2015) Fibrosis progression in nonalcoholic fatty liver vs nonalcoholic steatohepatitis: a systematic review and meta-analysis of paired-biopsy studies. Clin Gastroenterol Hepatol: Off Clin Pract J Am Gastroenterol Assoc 13:643-654 e641-649, quiz e639-640

26. McPherson S, Hardy T, Henderson E, Burt AD, Day CP, Anstee QM (2015) Evidence of NAFLD progression from steatosis to fibrosing-steatohepatitis using paired biopsies: implications for prognosis and clinical management. J Hepatol 62:1148-1155

27. Pais R, Charlotte F, Fedchuk L, et al. (2013) A systematic review of follow-up biopsies reveals disease progression in patients with nonalcoholic fatty liver. J Hepatol 59:550-556
28. Wong VW, Wong GL, Choi PC et al (2010) Disease progression of non-alcoholic fatty liver disease: a prospective study with paired liver biopsies at 3 years. Gut 59:969-974

29. Calle EE, Rodriguez C, Walker-Thurmond K, Thun MJ (2003) Overweight, obesity, and mortality from cancer in a prospectively studied cohort of U.S. adults. N Engl J Med 348:1625-1638

30. Margini C, Dufour JF (2015) The story of HCC in NAFLD: from epidemiology, across pathogenesis, to prevention and treatment. Liver Int 36:317-324

31. Yopp AC, Choti MA (2015) Non-alcoholic steatohepatitis-related hepatocellular carcinoma: a growing epidemic? Dig Dis 33:642647

32. Setiawan VW, Lim U, Lipworth L, et al. (2015) Sex and ethnic differences in the association of obesity with risk of hepatocellular carcinoma. Clin Gastroenterol Hepatol 14:309-316

33. Turati F, Talamini R, Pelucchi C et al (2013) Metabolic syndrome and hepatocellular carcinoma risk. Br J Cancer 108:222-228

34. Pocha C, Kolly P, Dufour JF (2015) Nonalcoholic fatty liver disease-related hepatocellular carcinoma: a problem of growing magnitude. Semin Liver Dis 35:304-317

35. Dyson J, Jaques B, Chattopadyhay D et al (2014) Hepatocellular cancer: the impact of obesity, type 2 diabetes and a multidisciplinary team. J Hepatol 60:110-117

36. Perumpail RB, Wong RJ, Ahmed A, Harrison SA (2015) Hepatocellular carcinoma in the setting of non-cirrhotic nonalcoholic fatty liver disease and the metabolic syndrome: US experience. Dig Dis Sci 60:3142-3148

37. Yki-Jarvinen H (2015) Nutritional modulation of non-alcoholic fatty liver disease and insulin resistance. Nutrients 7:9127-9138

38. Ziol M, Handra-Luca A, Kettaneh A et al (2005) Noninvasive assessment of liver fibrosis by measurement of stiffness in patients with chronic hepatitis C. Hepatology 41:48-54

39. Kotronen A, Peltonen M, Hakkarainen A et al (2009) Prediction of non-alcoholic fatty liver disease and liver fat using metabolic and genetic factors. Gastroenterology 137:865-872

40. Kotronen A, Westerbacka J, Bergholm R, Pietilainen KH, YkiJarvinen H (2007) Liver fat in the metabolic syndrome. J Clin Endocrinol Metab 92:3490-3497

41. Pacifico L, Ferraro F, Bonci E, Anania C, Romaggioli S, Chiesa C (2013) Upper limit of normal for alanine aminotransferase: quo vadis? Clin Chim Acta 422:29-39

42. Williams AL, Hoofnagle JH (1988) Ratio of serum aspartate to alanine aminotransferase in chronic hepatitis. Relationship to cirrhosis. Gastroenterology 95:734-739

43. Clark JM, Brancati FL, Diehl AM (2003) The prevalence and etiology of elevated aminotransferase levels in the United States. Am J Gastroenterol 98:960-967

44. Jenkins CM, Mancuso DJ, Yan W, Sims HF, Gibson B, Gross RW (2004) Identification, cloning, expression, and purification of three novel human calcium-independent phospholipase A2 family members possessing triacylglycerol lipase and acylglycerol transacylase activities. J Biol Chem 279:48968-48975

45. He S, McPhaul C, Li JZ et al (2010) A sequence variation (I148M) in PNPLA3 associated with nonalcoholic fatty liver disease disrupts triglyceride hydrolysis. J Biol Chem 285:6706-6715

46. Kumari M, Schoiswohl G, Chitraju C et al (2012) Adiponutrin functions as a nutritionally regulated lysophosphatidic acid acyltransferase. Cell Metab 15:691-702

47. Luukkonen P, Zhou Y, Sadevirta S, Leivonen M, Arola J, Oresic M, HyotylainenT, Yki-Jarvinen H (2016) Hepatic ceramides dissociate steatosis and insulin resistance in patients with non-alcoholic fatty liver disease. J Hepatol. doi:10.1016/j.jhep.2016.01.002

48. Kozlitina J, Smagris E, Stender S, et al. (2014) Exome-wide association study identifies a TM6SF2 variant that confers susceptibility to nonalcoholic fatty liver disease. Nat Genet 46:352-356 
49. Mahdessian H, Taxiarchis A, Popov S et al (2014) TM6SF2 is a regulator of liver fat metabolism influencing triglyceride secretion and hepatic lipid droplet content. Proc Natl Acad Sci U S A 111: 8913-8918

50. Sookoian S, Castano GO, Scian R et al (2015) Genetic variation in transmembrane 6 superfamily member 2 and the risk of nonalcoholic fatty liver disease and histological disease severity. Hepatology 61:515-525

51. Dongiovanni P, Petta S, Maglio C et al (2015) Transmembrane 6 superfamily member 2 gene variant disentangles nonalcoholic steatohepatitis from cardiovascular disease. Hepatology 61:506514

52. Falleti E, Cussigh A, Cmet S, Fabris C, Toniutto P (2016) PNPLA3 rs738409 and TM6SF2 rs58542926 variants increase the risk of hepatocellular carcinoma in alcoholic cirrhosis. Dig Liver Dis 48: 69-75

53. Zhou Y, Llaurado G, Oresic M, Hyotylainen T, Orho-Melander M, Yki-Jarvinen H (2015) Circulating triacylglycerol signatures and insulin sensitivity in NAFLD associated with the E167K variant in TM6SF2. J Hepatol 62:657-663 